\title{
Don't See the Dwellings for the Trees: Quantifying the Effect of Tree Growth on Multi-temporal Dwelling Extraction in a Refugee Camp
}

\author{
Fritjof Lüthje, Dirk Tiede and Petra Füreder \\ Department of Geoinformatics - Z_GIS, University of Salzburg/Austria·fritjof.luethje@sbg.ac.at
}

Full paper double blind review

\begin{abstract}
Automated and visual approaches for the monitoring of refugee or IDP camps based on satellite data are very important as independent information sources, especially for insecure and remote areas. Nevertheless, monitoring based on satellite data always has a certain degree of uncertainty, e.g. due to data quality, complexity of the area of investigation, seasonal pheonological problems, or algorithmic limitations. Within this paper, we aim to quantify one of these limiting aspects: the factor of vegetation (i.e. tree) growth and its effect on multi-temporal dwelling monitoring, hampering the identification of dwellings on the ground. For the refugee camp Djabal, Chad, we found that 2506 dwellings $(25 \%)$ of 2010 are at least partly affected by tree growth three years later (2013), which is influencing automated extraction methods, as well as visual interpretations. 395 of these dwellings were completely covered by vegetation and vegetation shadow, and were therefore not detectable anymore. Taking this factor into account, the decrease of dwellings between 2010 and 2013 is potentially lowered from $10 \%$ to $5 \%$.
\end{abstract}

\section{Introduction}

With the increased availability of very high resolution (VHR) satellite data, automated or visual dwelling extractions for monitoring the development of refugee camps or camps for internally displaced people (IDP) offers new possibilities for aid agencies. Especially for insecure and remote areas, independent information sources are of great importance. Automated methods are often limited in their spectrum of application, or vice versa highly tailored for one use-case (VOIGT et al. 2014), but visual interpretation is also often hampered by the complexity and size of the camps (FÜREDER et al. 2014). Depending on the circumstances and the data availability, different approaches with different degrees of reliability are commonly used (SPRÖHNLE et al. 2014). Inherent to all these approaches (visual and automated) is a degree of uncertainty concerning the robustness of the dwelling extractions: on satellite images, dwellings covered with white plastic sheeting may appear very bright in the days after a rainfall, but will hardly be distinguished from the ground after a sandstorm. In respect to camp monitoring, different points in time represent different factors influencing the reliability of the results such as different seasons of the image acquisition, changes in shelter type within the camp, or vegetation growth within the camp.

SPRÖHNLE et al. (2014) showed a comparison of different algorithms and visual interpretations and their robustness in regard of camp complexity. Within this paper, we aim to

GI_Forum - Journal for Geographic Information Science, 1-2015.

(C) Herbert Wichmann Verlag, VDE VERLAG GMBH, Berlin/Offenbach. ISBN 978-3-87907-558-4.

(C) ÖAW Verlag, Wien. ISSN 2308-1708, doi:10.1553/giscience2015s406. 
quantify the factor of vegetation (i.e. tree) growth and its effect on multi-temporal dwelling monitoring, hampering the identification of dwellings on the ground. This work is part of a monitoring study within the EU FP7-project G-SEXTANT (Geospatial services in support of EU external action), aimed at identifying indicators for repatriation or stabilization of structures in the refugee camp Djabal. The camp is located in eastern Chad, directly to the west of the town Goz Beida. The camp exists since 2003, and accommodates several thousands of refugees, mainly from the Sudanese conflict of 2002. The camp is managed by the UNHCR and organized in communal zones (ENOUGHPROJECT 2009).

The environment is mainly characterized by savannah, with sparse to dense shrubs and tree stands. Within the camp area, single trees were reportedly planted soon after the camp was created to provide shade and relief from the hot sun (STANDNOW 2008).

Analyses based on multi-temporal VHR satellite imagery for monitoring the development of dwellings and the growth of single trees (as indicators for stabilized living conditions) within the camp, revealed the question of an interrelationship between tree growth, i.e. the vertical vegetation projective cover (VPC) (LU et al. 2003), and disappearing or hidden dwellings. Within this paper, we try to quantify the effect of tree growth on the detectability of the dwellings underneath in an automated camp monitoring process.

\section{Input Data and Data Processing}

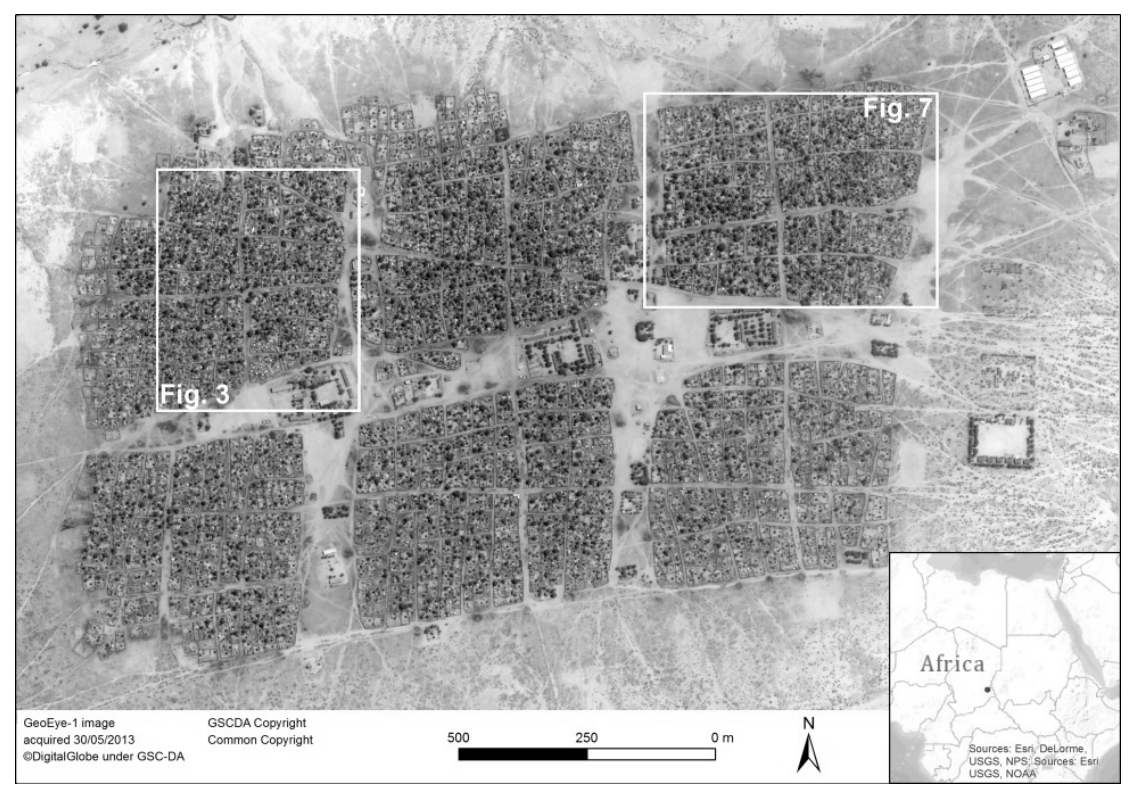

Fig. 1: The Djabal refugee camp (GeoEye-1 image, 30/05/2013) in eastern Chad, near the border region to western Sudan and the Darfur region. The image is shown in false-colour combination (4/3/2) to highlight the vegetation within the refugee camp area (red tones). The location of the subsets of Fig. 3 and Fig. 7 are indicated as white boxes. 
We used VHR images from three different points in time to monitor the development of dwellings as well as single trees within the camp. The first satellite image from June $21^{\text {st }}$, 2007 was taken by the QuickBird satellite. Orthorectified and pan-sharpened using the IHSmethod, this image was used as basis to co-register the two more recent images. The intermediate satellite image from March 31 $1^{\text {st }}, 2010$ was collected by the GeoEye- 1 satellite, and also pan-sharpened using the IHS method. The most recent satellite image, acquired on May $30^{\text {th }}$, 2013 by the GeoEye-1 satellite (cf. Fig. 1), was atmospherically corrected using the Catena workflow at DLR (KRAUß et al. 2013) and pan-sharpened with the HCS method.

All images were gathered after the rainy season. Weeds and small shrubs are dried out, but the trees still have a strong vital signal in the near infrared band. This fact enables a fast detection of a vegetation mask, and the subsequent extraction of single trees.

\section{Methodology}

In the following chapter, the approaches for dwelling extractions as well as single tree extractions are described (cf. Fig. 2). All approaches utilize object-based image analysis (OBIA, BLASCHKE 2010) techniques, and are implemented using Cognition Network Language (CNL) within the eCognition development environment (Trimble Geospatial).

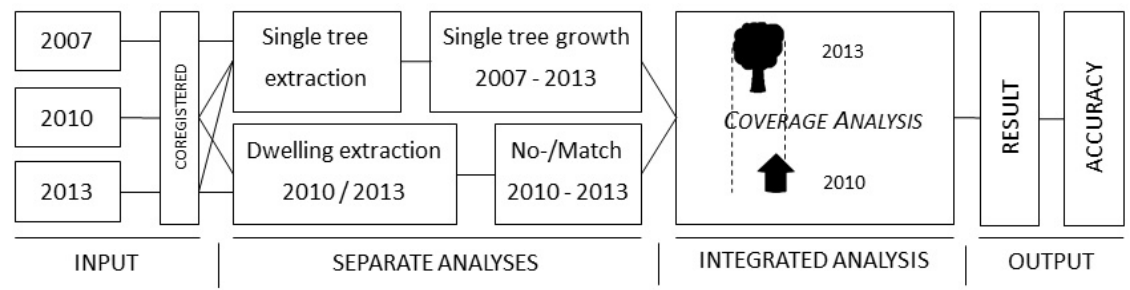

Fig. 2: Workflow for dwelling and single tree extraction, and subsequent coverage analysis of the two separate intermediate results from multi-temporal satellite images

As a final step, results of the dwelling extractions and the single tree extractions for 2010 and 2013 are analysed by an object-specific geographic overlay, to identify dwellings which are affected by VPC. The degree to which the classification results are affected by hidden dwellings is quantified.

\subsection{Dwelling Extraction}

Dwelling extraction was conducted for all three points in time; within this study, only classifications from 2010 and 2013 are taken into account.

For the analyses of the image from 2010, a so-called master ruleset (TIEDE et al. 2010b) was adapted, which was developed and applied for various IDP camps in Darfur and aimed at minimising the effort of transferability. Three different types of dwellings were thereby separated: bright dwellings (mainly tents), dark dwellings (mainly traditional round huts), 
and large tents indicating supply areas. The extraction of dark dwellings was hampered due to other dark structures like fences, often attached to the dwellings. Overall 10,311 dwellings were extracted: 3,573 bright dwellings, 6,623 dark dwellings, and 115 large tents.

The dwelling extraction performed for the image of 2013 is based on a new approach that makes use of a template matching technique within an object-based environment. In order to avoid misclassifications caused by vegetation, an initial vegetation mask was created based on an NDVI threshold of 0.3. In a first sequence, the dwelling extraction focuses on the detection of well identifiable objects (i.e. clearly defined dwellings) for the respective class, using spectral differences between the objects of interest and their adjacent environment. The same classes were identified as for the 2010 image: traditional round huts which are darker than the adjacent reddish soil, bright dwellings (mainly tents), and larger structures (indicating supply areas), both brighter than the adjacent reddish soil. This subset of objects was used as set of samples to generate templates for each class, which were applied to the whole image. Thereby, the classification could be improved by a considerable number of objects matching the templates. Henceforth, the detected features are refined using geometrical as well as hierarchical features (TIEDE et al. 2010a). A total number of 9,127 dwellings were extracted for the image of 2013. These numbers were independently verified within the project (by different project partners) showing sufficient accuracies and the results (products) were positively validated against the user requirements.

By only comparing the bare number of dwellings, a change or decrease of almost 1200 dwellings seems to have taken place, representing a decrease of more than ten percent. In the following chapter, we investigate how the number of trees and tree growth during the years potentially influences the automated extraction of dwellings.

\subsection{Single Tree Extraction}

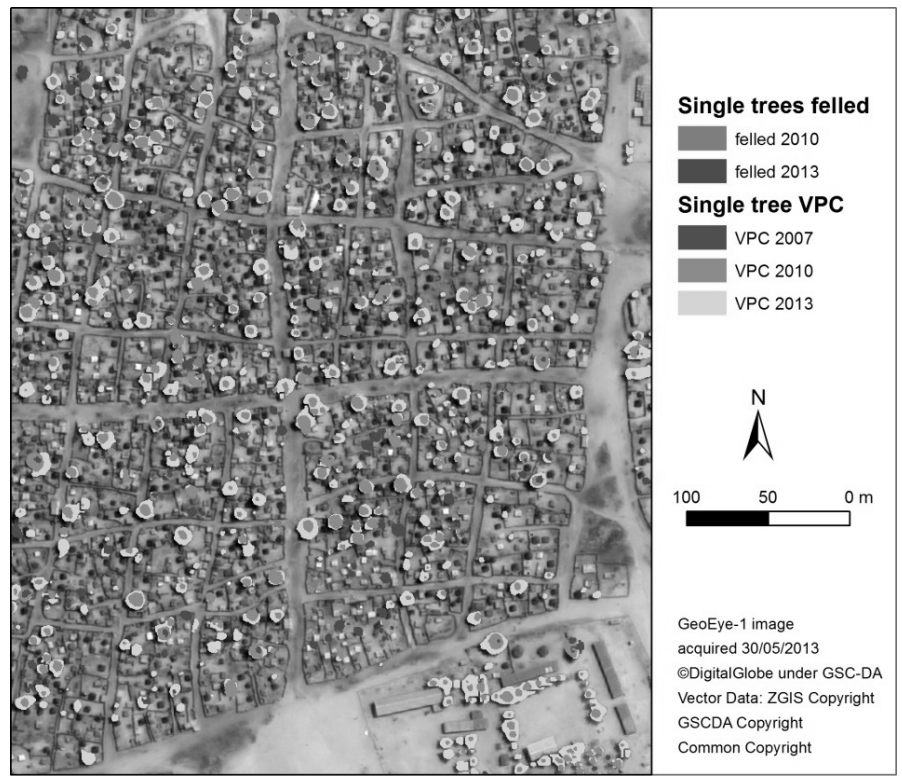

Fig. 3:

Tri-annual rings of VPC-growth: dark green shows the extent of VPC as of 2007, mid-green the intermediate extent of VPC as of 2010, and light green depicts the most recent extent of VPC as of 2013. The orange polygons represent trees that have been felled between 2007 and 2010; the red polygons represent those that have been felled between 2010 and 2013. 
The VPC values of all three different points in time (2007/2010/2013) were derived using a three-step approach: (1) a vegetation mask was applied, which is based on the NDVI (see previous chapter). (2) A local maxima detection focusing on spectral maxima was applied within the vegetation mask to identify single tree crown-top objects, assuming that higher parts of the trees are regions of high reflectance (cf. WULDER et al. 2000). (3) A regiongrowing algorithm using spectral information as constraints was implemented to delineate the single tree and thus the VPC. The different results for all three years were combined to reveal the different vegetation stages and the growth of each single tree. A total number of 3,359 single trees was detected for 2013, while 586 were removed at that time. The results are depicted in Fig. 3 in different shades of green, while eliminated trees are depicted in shades of red. The tree extraction revealed a high increase of single trees between 2007 and 2010, whereas the tree coverage shows an extensive growth between 2010 and 2013 (cf. Fig. 4).

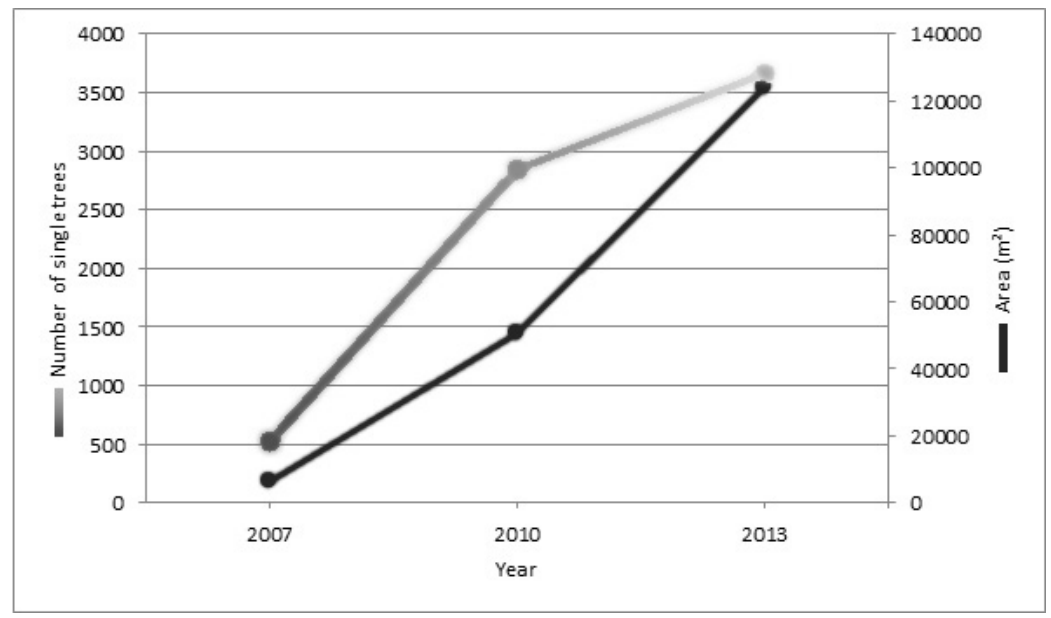

Fig. 4: Development of single tree numbers (green line where dark green stands for 2007, mid-green for 2010, and bright green for 2013), as well as tree coverage (in $\mathrm{m}^{2}$, black line) from 2007 to 2010 to 2013

\subsection{Geographic Overlay and Object-by-Object Comparison}

In the course of the dwelling extraction for 2013, and the subsequent comparison with the dwellings extracted from 2010, trees and the extensive growth in between these two points in time were identified as a potential influencing factor regarding dwelling extraction accuracy. For the purpose of quantifying the effect of VPC growth on hidden or partly covered dwellings, the dwelling extractions as well as the single tree VPCs from 2010 and 2013 were compared (cf. Fig. 5). A geographic overlay of both datasets was conducted within the OBIA software environment, making use of the data model's inherent topological relationships for detailed object-by-object comparison (cf. TIEDE 2014). The workflow is described in the following paragraph. 


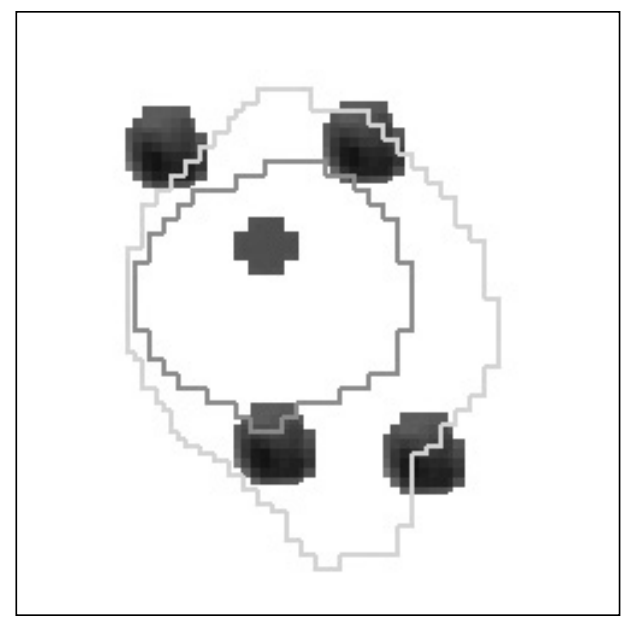

Fig. 5:

Modelled VPC development of a single tree over time for four different dwellings. The green lines represent the tree growth for the three different points in time (dark green for 2007, mid-green for 2010, and bright green for 2013). The lower-left dwelling is totally covered in 2013; the upper right dwelling is strongly covered; the lower-right dwelling is covered by $50 \%$; the upper-left dwelling is only partially affected.

It is assumed that only the upper-left and the lower right dwellings are still detectable in 2013.

In a first step, all dwellings from 2010, which are affected by VPC from 2013, have been selected. From these dwellings, all dwellings with a corresponding dwelling in 2013 were removed. Next, the remaining dwellings from 2010 were categorized according to their percentage of tree coverage in 2013. In parallel, those dwellings from 2010, which are affected by VPC of 2013 and have a corresponding dwelling in 2013, are also categorized according to the same range of percentages. Thus, the effect of tree growth could be evaluated for both the dwellings that are affected by VPC and still could be detected on both images on the one hand, and those dwellings that were not detected anymore in 2010 or 2013 on the other hand (either as they are hidden by vegetation, have been removed, or were simply missed by the extraction approach).

\subsection{Accuracy Assessment}

The accuracy of the analysis was examined by dividing the camp area into a regular grid. From this grid, 25 cells were randomly chosen (cf. Fig. 8, right). Subsequently, the dwellings from 2010 with any vegetation coverage but no corresponding dwellings in 2013 were visually assessed. By categorizing the dwellings into three classes (still existing, no longer existing, and completely covered) and taking into account their original class of vegetation cover percentage, a statement about the actual effect of VPC on the detectability of dwellings could be provided.

\section{Results}

A total number of 2506 dwellings from the dwelling extraction of 2010 were affected by tree coverage of 2013. This is almost 25 percent of all dwellings from 2010. From these 2506 dwellings, 1903 dwellings did not have a corresponding dwelling in 2013, while 603 dwellings had a corresponding dwelling. The dwellings from these two categories were then covered by trees from 2013, and assigned to one of five coverage classes $(20 \%$ each). The distribution of values is shown in Fig. 6. 


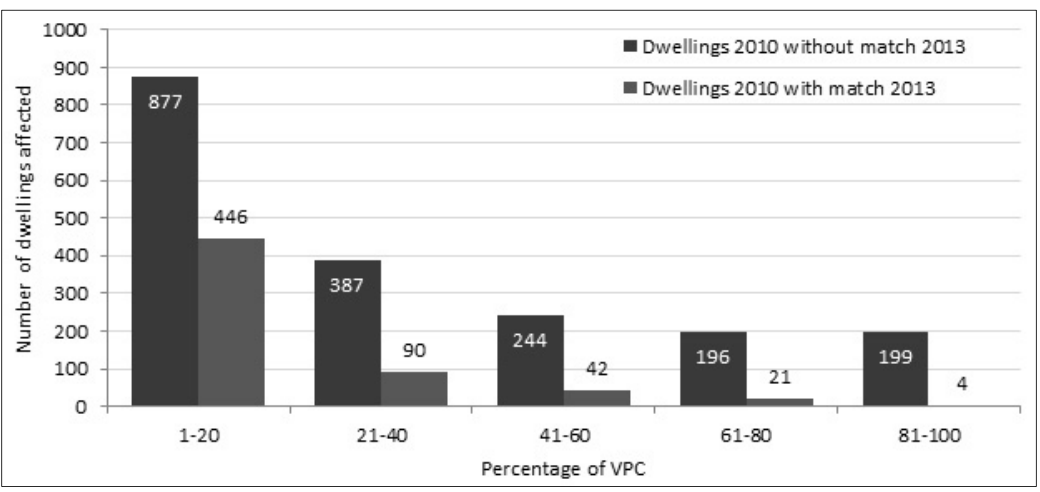

Fig. 6: Number of dwellings affected by different degree of VPC coverage. Red: Number of dwellings 2010 affected by VPC in 2013 with no corresponding dwelling in 2013; Median of $37 \%$. Blue: Number of dwellings 2010 affected by VPC in 2013 with a corresponding dwelling in 2013; Median of $19 \%$.

To visualize the bare numbers of Fig. 6, a subset of the Djabal refugee camp with the classification according to the coverage percent classes is shown in Fig. 7. Here, the distinction into dwellings 2010 with a matching dwelling in 2013, and without a matching dwelling in 2013, is also depicted. Almost all cases of coverage and detection can be found in this subset: Dwellings that are hardly covered by a tree from 2013 and could be detected in 2013 as well. Dwellings belonging to the class $21-40 \%$ coverage, but were still found in the classification of 2013. Other dwellings belong to the class $21-40 \%$ coverage, but were not found in 2013 anymore. For the classes with equal or higher coverage than $61 \%$, most dwellings from 2010 did not have a corresponding dwelling in 2013. This is in line with the values shown in Fig. 4.

The accuracy assessment was carried out as described in the respective chapter above. The random sampling resulted in 107 dwellings in 25 grid cells, which met the described accuracy assessment criteria, and which were assessed visually (cf. Fig. 8). The class 'nonexisting' indicates that a dwelling from 2010 with tree coverage in 2013 does not exist anymore; vice versa, the class 'existing' represents those dwellings that are still - at least partly - visible in 2013. The class 'covered' is of special interest, since these are dwellings that cannot be identified in 2013. No statement about their existence can be made, reasons for this is to a high degree the overlapping vegetation, but also additional effects like shadows casted by the trees. This clearly indicates that tree coverage and resulting tree shadows are important factors for automated dwelling extraction if vegetation coverage is high. The lower coverage classes are, however, not affected due to the low degree of overlap, which also reduces the shadow effect. Within the coverage classes $1-20 \%$ and $21-40 \%$ there was no dwelling completely non-visible in 2013; either they were removed or not detected by the automated approach in 2013. Only if the percentage of vegetation coverage increases, the effect is of interest for the dwelling detection.

On the other hand, it is also obvious that a considerable number of dwellings were not detected by the automated approach, although they are still visible in 2013. This is especially true for dwellings in the lowest coverage class, where $25 \%$ of the dwellings were missed by the automated approach. 


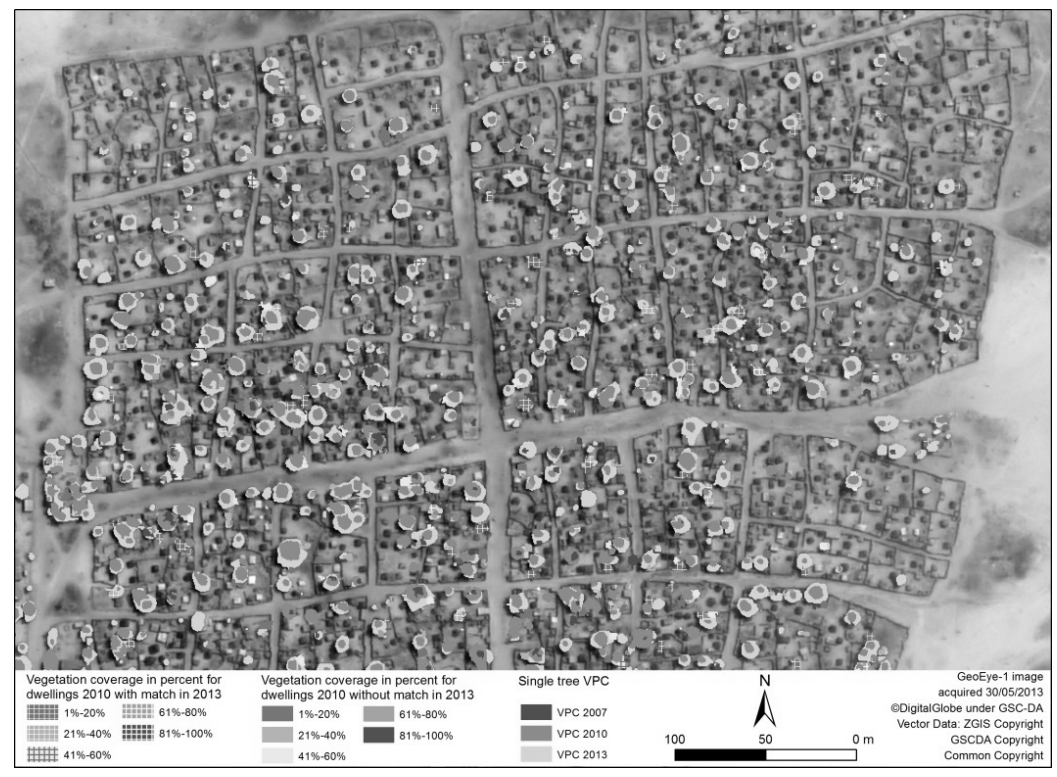

Fig. 7: Classification of dwellings from 2010 according to existence and non-existence of corresponding dwellings in 2013 and according to degree of vegetation coverage by single trees.
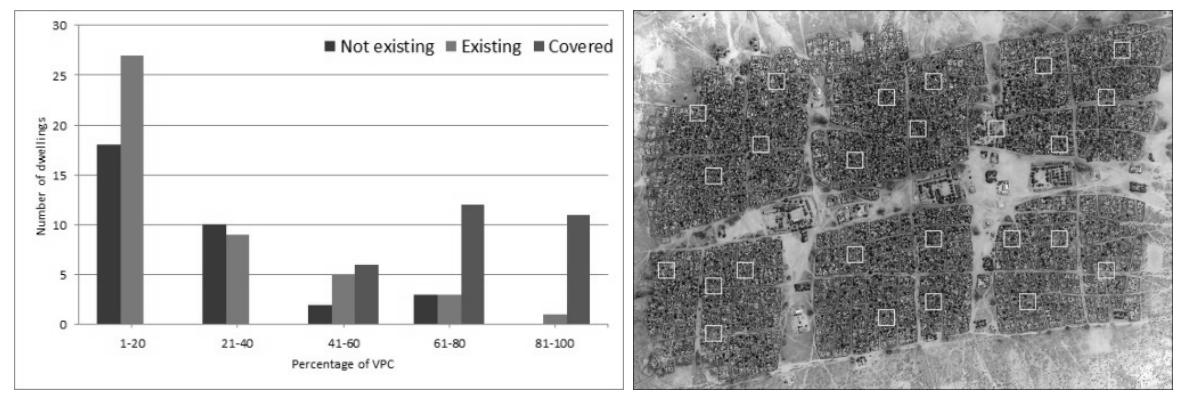

Fig. 8: Left: Accuracy of tree-covered dwellings within 25 random sampling grid cells; assessed visually and distinguished according to VPC classes. Red pillars represent non-existing dwellings, blue pillars stand for still existing dwellings, green pillars show totally covered dwellings. Right: Distribution of the 25 grid cells in the camp area.

Within that class, the effect of classification errors is more severe than the effect of vegetation coverage. The reason for this is linked to the object-based dwelling detection approaches: Since the presented approaches use form and size of the delineated objects for the classification, partly overlapped dwellings are hard to be classified. In the upper classes, i.e. $61-80 \%$ and $81-100 \%$ coverage, the effect of coverage is highly dominant. The intermediate class of $41-60 \%$ should be regarded as the transitional class, where the effect of vegetation coverage also plays an important, yet not dominant role. 


\section{Conclusion and Outlook}

In this paper, we presented an approach to quantify the effect of tree growth on automated dwelling extractions from VHR satellite imagery in a refugee camp. This is an especially important factor for camps where trees are planted on purpose, e.g. for the provision of shade, where a fast tree crown growth is intended.

The Djabal refugee camp is a good example for monitoring this effect. Shortly after establishing the camp, the UNHCR started planting fast growing trees to provide shade and relief from the sun (STANDNOW 2008). The growth of these trees can be clearly observed on the VHR satellite images. The effect of tree growth and VPC that occludes dwellings is of special interest in regard to monitoring the camp development.

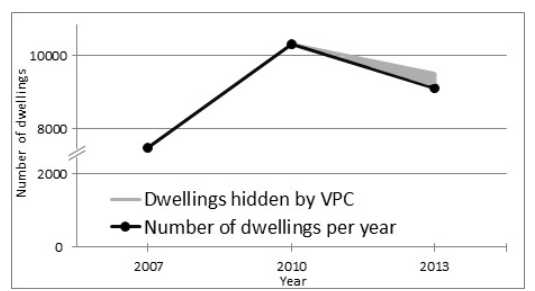

Fig. 9:

Development of the number of dwellings derived from the respective classification per year (black) and the additional span that is opened up by adding those dwellings that are strongly VPC-influenced and thereby not detectable any more.

The analyses in this paper have shown that at least 2506 dwellings are affected by tree coverage. This influences the automated extraction methods, which rely on object-based methods and are not tailored to only partly visible dwellings. As an outcome of the analyses and the accuracy assessment, we propose to adapt - as far as possible - automated analyses to also detect dwellings partly overlapped by tree crowns and/or to take the factor of VPC into account for multi-temporal monitoring and change detection approaches. For the categories of $61 \%$ coverage and upwards, dwellings should be added to the results of the newer analysis as potentially still existing. Since there were only very few corresponding dwellings found on the image of 2013, and the accuracy revealed that most of the dwellings are fully covered by vegetation and resulting shade, it can be assumed that these dwellings may still exist. The coverage class of 41-60\% can be regarded as a transitional class. By taking into account the dwellings of the two highest coverage classes, the total number of 9127 dwellings would increase by 395 to 9522 (cf. Fig. 9). For the overall monitoring, this would not imply a decrease of dwellings by ten percent in this case, but only by five percent, which makes a considerable difference.

Results of the approach have successfully passed the independent verification and validation procedure within the project context, and the validation team pronounced the usefulness of the investigation and information for the user. Hence, a quantification of the effect of tree growth for the dwelling extraction is regarded as an important consideration within the monitoring of refugee camps. Not taking into account the growth of trees and related coverage of a certain percentage of dwellings can result in a profound underestimation of dwellings, and of the affected population. There is of course no evidence if dwellings completely covered by trees/tree shadows are still there or have been removed in the meantime. Further investigations should focus on additional VHR imagery from the dry seasons to obtain more evidence in this respect, at least for the semi-deciduous trees. Nevertheless, even with a limited amount of VHR images, vegetation covered dwellings should be in- 
cluded as an uncertainty factor to show the range of dwelling extraction results, which can then be considered for further population estimations.

\section{Acknowledgements}

The research leading to these results has received funding from the European Union's Seventh Framework Program (FP7/2007-2013) under grant agreement $n^{\circ} 218822$, G-MOSAIC, and grant agreement $n^{\circ} 312703$, G-SEXTANT. We would like to thank the colleagues from the project partners EU SatCen (European Union Satellite Centre) and SRC (Space Research Centre) for conducting the verification and validation of the results.

\section{References}

BLASCHKE, T. (2010), Object based image analysis for remote sensing. ISPRS Journal of Photogrammetry and Remote Sensing, 65, 2-16.

ENOUGHPROJECT (2009), Scenes from Djabal Refugee Camp in Eastern Chad. http://www. enoughproject.org/blogs/scenes-djabal-refugee-camp-eastern-chad (28/01/2015).

FÜREDER, P., TIEDE, D., LÜTHJE, F. \& LANG, S. (2014), Object-based dwelling extraction in refugee/IDP camps - challenges in an operational mode. South-Eastern European Journal of Earth Observation and Geomatics, 3 (2S), 539-544.

Krauß T., D'Angelo P., Schneider M. \& Gstaiger V. (2013), The Fully Automatic Optical Processing System CATENA at DLR. ISPRS Int. Arch. Photogramm. Remote Sens. Spatial Inf. Sci, Vol. XL-1/W1, 177-181.

LU, H., RAUPACH, M., MCViCAR, T. \& BARRETT, D. (2003), Decomposition of vegetation cover into woody and herbaceous components using AVHRR NDVI time series. Remote Sensing of Environment, 86, 1-18.

StANDNOW (2008), Camp Djabal. http://www.standnow.org/files/file/standfast/ STANDFAST\%20camp\%20djabal.pdf (28/01/2015).

Spröhnle, K., Tiede, D., Schoepfer, E., Füreder, P., Svanberg, A. \& Rost, T. (2014), Earth Observation-Based Dwelling Detection Approaches in a Highly Complex Refugee Camp Environment - A Comparative Study. Remote Sensing, 6 (10), 9277-9297.

TIEDE, D. (2014), A new geospatial overlay method for the analysis and visualization of spatial change patterns using object-oriented data modelling concepts. Cartography and Geographic Information Science, 41, 227-234.

Tiede, D., LAng, S., Albrecht, F. \& Hölbling, D. (2010a), Object-based Class Modeling for Cadastre-constrained Delineation of Geo-objects. Photogrammetric Engineering Remote Sensing, 76, 193-202.

Tiede, D., LAnG, S., Hölbling, D. \& FÜreder, P. (2010b), Transferability of OBIA rulesets for IDP Camp Analysis in Darfur. In: AdDINK, E. A. \& CoIllie, F. M. B. VAN (Eds.), GEOBIA. ISPRS, Vol. XXXVIII-4/C7. Archives ISSN 1682-1777.

Voigt, S., Schoepfer, E., Fourie, C. \& MAger, A. (2014), Towards semi-automated satellite mapping for humanitarian situational awareness. In: Global Humanitarian Technology Conference (GHTC), 412-416.

Wulder, M., Niemann, K. O. \& Goodenough, D. G. (2000), Local Maximum Filtering for the Extraction of Tree Locations and Basal Area from High Spatial Resolution Imagery. Remote Sensing of Environment, 73, 103-114. 